\title{
A Roadmap to Robot Motion Planning Software Development
}

\author{
ALEXANDER PÉREZ, JAN ROSELL
}

Institute of Industrial and Control Engineering (IOC), Technical University of Catalonia (UPC), Av. Diagonal 647, 11 th floor, 08028 Barcelona, Spain

\begin{abstract}
PhD programs and graduate studies in robotics usually include motion planning among its main subjects. Students that focus their research in this subject find themselves trapped in the necessity of programming an environment where to test and validate their theoretic contributions. The programming of this robot motion planning environment is a big challenge. It requires on the one hand good programming skills involving the use of software development tools, programming paradigms, or the knowledge of computational complexity and efficiency issues. On the other hand it requires coping with different related issues like the modeling of objects, computational geometry problems and graphical representations and interfaces. The mastering of all these techniques is good for the curricula of roboticists with a motion planning profile. Nevertheless, the time and effort devoted to this end must remain reasonable. Within this framework, the aim of this paper is to provide the students with a roadmap to help them in the development of the software tools needed to test and validate their robot motion planners. The proposals are made within the scope of multi-platform open source code. (c) 2009 Wiley Periodicals, Inc. Comput Appl Eng Educ; Published online in Wiley InterScience (www.interscience.wiley.com); DOI 10.1002/cae.20269
\end{abstract}

Keywords: robotics; software development; path planning

\section{PROBLEM STATEMENT}

One of the main tasks in robotics is to plan collisionfree paths for a robot from a start to a goal configuration among the obstacles in the workspace. This problem is known as robot motion planning

Correspondence to J. Rosell (jan.rosell@upc.edu).

Contract grant sponsor: CICYT; Contract grant numbers: DPI2007-63665, DPI2008-02448.
[1-3]. The basic approach is a computationally hard geometric problem that can get much harder if other issues are to be taken into account like sensor uncertainties, differential constraints, or the existence of dynamic obstacles. Robot motion planners must cope with the modeling of objects and their location, with collision detection and other computational geometry problems, with graph representations and search algorithms, and with graphic rendering and other graphical user interface issues. Therefore, the development of a robot motion planner is not an easy 
task since it involves many different issues ranging from the use of software development tools to the knowledge of computational geometry solutions.

There are some open source motion planners available on the web like An Object-Oriented Programming System for Motion Planning [4] (OOPSMP, www.kavrakilab.org/software), the Motion Planning Kit (http://ai.stanford.edu/mitul/mpk) or the Motion Strategy Library (http://msl.cs.uiuc.edu/msl/). The OOPSMP is a path planner that currently allows solving problems for free-flying robots moving on a plane using the basic versions of either probabilistic roadmaps (PRM, [5]), or rapidly exploring random trees $(R R T,[6])$. It has the interesting didactic feature of allowing to consecutively execute all the steps of the planning process and showing, for instance, the robot at each of the sampled configurations. Although it is conceived to be flexible and modular (it is implemented using the generic programming paradigm based on XML descriptions), there is not enough documentation yet to allow its use for programming and testing new algorithms or variants. The $M S L$ and the $M P K$ are, on the other hand, basically conceived as tools to prove and illustrate the motion planners developed by their authors, namely the basic $R R T \mathrm{~s}$ and all its variants $(M S L)$ and the $S B L$ algorithm, a fast single-query probabilistic roadmap path planner $(M P K)$. They also allow dealing with more complex problems involving robot manipulators ( $M P K$ also includes a multirobot module), or mobile robots with kinematic constraints (for the case of $M S L$ ).

Although all these planners have interesting features, they do not cover the needs from a teaching perspective: They are not easy to use and it is hard to get an insight into their structure in order to adapt and extend them to the particular needs. This is the reason why, in order to have an environment where to test and validate theoretic contributions in motion planning, it ends up being more efficient to build a planner from scratch. From the learning point of view, in the scope of $\mathrm{PhD}$ programs and graduate studies in robotics, this is not a bad issue, since it gives the students the opportunity to face different problems and to acquire a global view. Nevertheless, some guidelines are always welcome in order to keep the effort reasonably bounded. This paper makes a proposal of some useful tools (and illustrates how to use them) with the aim of paving the path towards the development of motion planning software.

The paper is structured as follows. Tools Section presents the set of tools with which the students must get involved in the development of robot motion planning software. They are building bricks that cover from general software development tools, operating systems and compliers to graphics, GUIs and computational geometry libraries. Certainly many different alternatives do exist for each of them. The proposal of this paper is made having in mind an open source philosophy and a multi-platform approach, and is currently being used with success at the Technical University of Catalonia to teach "Planning in robotics" within the PhD Program "Automatic Control, Robotics and Computer Vision (ARV)". After the tools' overview, Kick-Off Examples Section presents some kick-off examples to facilitate the students' start-up. The examples code can be downloaded from http://iocnet.upc.edu/usuaris/JanRosell// EducationalTools.html.

\section{TOOLS}

\section{Software Development Tools}

When a new software project begins, it is necessary to define the tools to be used all along the process. Which programming techniques will be used? How will a design be described? How will the development history and backups of the project be maintained? A motion planning software is not a small application; it really is a big software project where many libraries are working together. For this reason, it is important to begin with a good task plan covering all the development steps.

One of the most published programming paradigms in the last decade is the Object Oriented Programming (OOP, [7]). Through the $O O P$ it is possible to comfortably work with the models (objects) of all the actors involved in the problem, like the configuration space, the workspace, the obstacles and the robots for the case of motion planning problems. $O O P$ may be seen as a collection of cooperating objects, as opposed to a traditional view in which a program may be seen as a group of procedures. Every type of object in a $O O P$ application is defined by a class. In $O O P$, each object is capable of receiving messages, processing data, and sending messages to other objects. $O O P$ allows the creation of modular and reusable code.

Closer to the OOP methodology, grows the Unified Modeling Language that is a meta language used to graphically show a software design [8]. Documentation about this meta language is regularly posted in the Object Group website (www.uml.org). Using an $U M L$ graphic tool like StarUML (www. staruml.com) it is possible to make an initial global design at class level, that can later be refined or modified (Fig. 1). One advantage of using the 


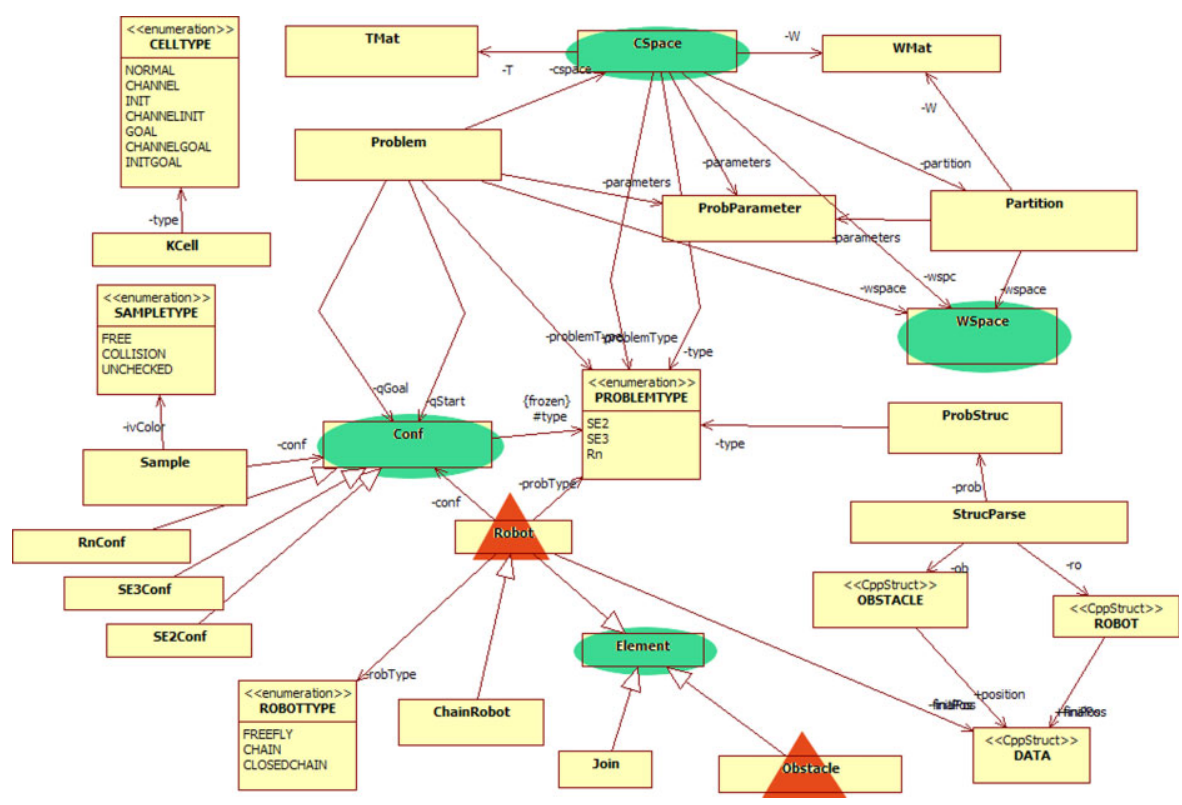

Figure 1 The UML model of a motion planner project where the main classes (highlighted in green) represent the workspace, the configuration space, the configurations and the geometric elements (implemented in the derived classes representing obstacles and the robot, highlighted in red). [Color figure can be viewed in the online issue, which is available at www.interscience. wiley.com.]

StarUML over other tools is its ability to generate the source and header files (*.cpp and *.h files, respectively) for all the associated classes in the design. Furthermore it can, in a process called reverse engineering, extract a graph of the class hierarchy from all the project files.

All the steps in a long software developing process must be stored for tracking and debugging purposes. A good way of doing this is through a control version system like Subversion SVN (https:// subversion.tigris.org). These systems provide a good environment to store and restore any possible version of each file in the project all along the development time, and it is very useful to manage projects involving several programmers at the same time. Other tools like CVS (www.nongnu.org/cvs) or a commercial solution like SafeSource (http://msdn. microsoft.com/en-us/vs2005/aa718670.aspx), can be used, but $S V N$ is currently the preferred control version system in the open source community. It provides a set of tools for common administrative server tasks and client operations. For a complete reference on $S V N$, see Ref. [9].

An effective and easy solution of the server side is OpenSVN (https://opensvn.csie.org), it is an opensource initiative for open-source projects. It provides a good access and availability and it can store a large amount of files. For the client side, TortoiseSVN (http://tortoisesvn.net) is a Windows shell extension which integrates into Windows Explorer. For Linux environments, kdesvn (http://kdesvn.alwins-world.de) is a high integrated application for KDesktop. Similar tools can be found for other Linux desktops.

These tools can be complemented by many others like those that allow the comparing of two versions of the same file to track and revise the changes (e.g., winmerge at www.winmerge.org, KDiff3 at http:// kdiff3.sourceforge.net or Meld at http://meld.source forge.net).

Finally, a good programmer should properly document the code. This can easily be done using Doxygen (www.doxygen.org), a multi-platform documentation system for several languages like $\mathrm{C}++$ and java. By only adding some comments within the code with a special mark, this software automatically generates a great documentation.

\section{O.S. and Compilers}

Usually, the main programming language used for this kind of applications is $\mathrm{C}++$. It is desirable that the software be multi-platform, in other words that it can either run in Windows or Linux. To achieve this purpose, it is necessary to use for each platform its own compiler: the $c l$ compiler can be used on Windows and gcc (http://gcc.gnu.org) on Linux. The $\mathrm{cl}$ compiler can be obtained from them Microsoft web 
site when installing any license type of Visual Studio, including the free license Express Edition (www.microsoft.com/express/download). Several other $\mathrm{C}++$ compilers can be found on Internet from many other sources like HP, Borland or Intel and they can be used in Windows and/or Linux, although the use of system compliers is preferred.

The $g c c$ and $c l$ compilers are command line, and the process to build the applications can be a bit thorny. For this reason, it is useful to use another program to manage the compilation and linkage processes. This application in "nix" environments is called Make; in Windows the analogous application is called nmake. These two applications do not describe the process in the same way. The solution to this problem is CMake (www.cmake.org), an open-source multi-platform make system. CMake has a GUI wizard in Windows (Fig. 2) that helps to configure the process. A complete reference for CMake is available in Ref. [10]. The CMake utility allows the user to prepare the project in both Windows and Linux, relieving him/her from the task of configuring the project for each platform. This helps the applications to be really multi-platform since the projects for compiling and linking them are easily obtained for each platform from the instructions coded using a script language in a text file called CmakeList.txt.

Each programmer has its own preferences for the Integrated Developer Environment to be used to write and debug the code in a friendly way. IDEs like Visual Studio on Windows or KDevelop on Linux offer many facilities to speed up the programming process (Fig. 3), but each one has its own particularities in the project description and management. In Windows,
CMake can configure the project file for a particular version of Visual Studio or create a descriptive file to be processed with nmake. In Linux, CMake can create a descriptive file called Makefile and in a future version it will configure the project for KDevelop.

\section{Graphic Tools}

Graphics. The graphic interface to show threedimensional models can be build using Coin $3 D$ (www.coin3d.org). This graphics library is based on OpenInventor sources delivered from Silicon Graphics and it is currently the implementation adopted for all Debian Linux distributions. Coin $3 D$ provides an interface similar to a $C A D$ software. It can manage either basic models like spheres, cones and cubes, or general forms modeled with triangular meshes (Fig. 4). Furthermore it is multi-platform and therefore it can also compile and run on Windows. It can be installed from a binary distributions repository or compiled from the source code. Coin $3 D$ must work together with a GUI library, that can be either $Q t$, Motif/Xt, Windows or MacOs APIs. For this interaction, SoQt or Quater, SoXt, SoWin, and Sc21 libraries are, respectively, required.

Any three-dimensional model can be described as a text file through Inventor or VRML languages (http:// www.w3.org/MarkUp/VRML). They describe a scene graph with high level objects like cubes, cylinders, cones or spheres or any grouping of these, as well as triangular meshes. Also, property nodes are introduced to affect the way shapes are drawn. Coin $3 D$ can load any scene described with either Inventor or $V R M L$ files.

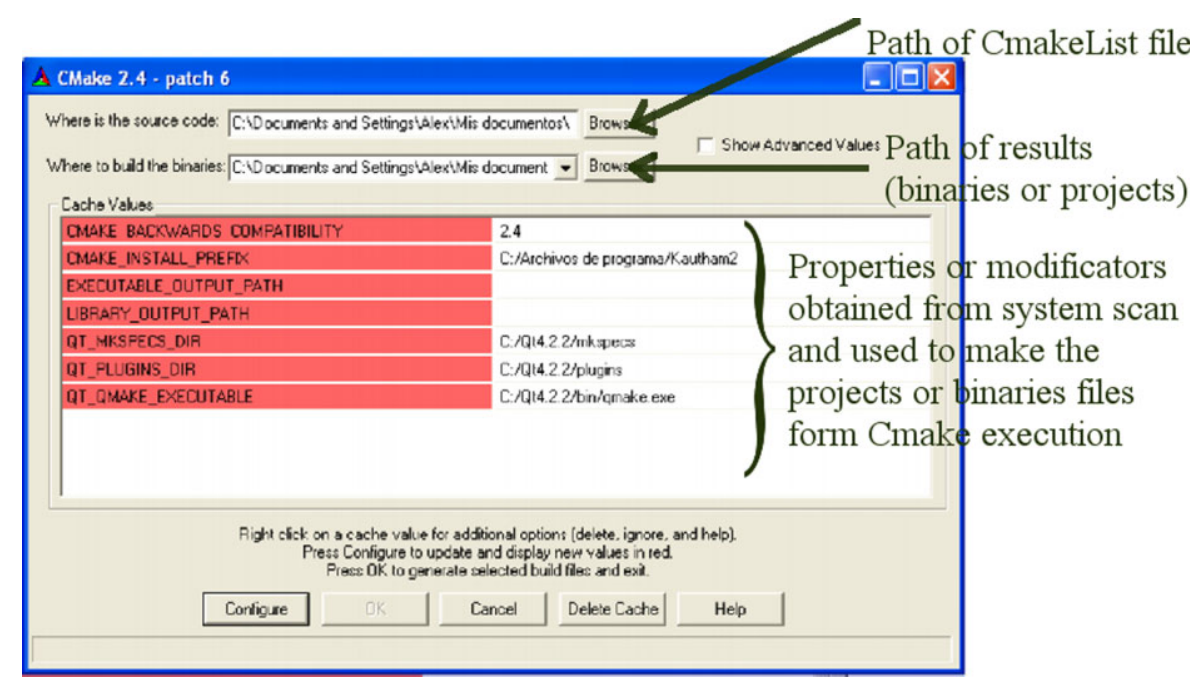

Figure 2 CMake GUI in Windows. [Color figure can be viewed in the online issue, which is available at www.interscience.wiley.com.] 


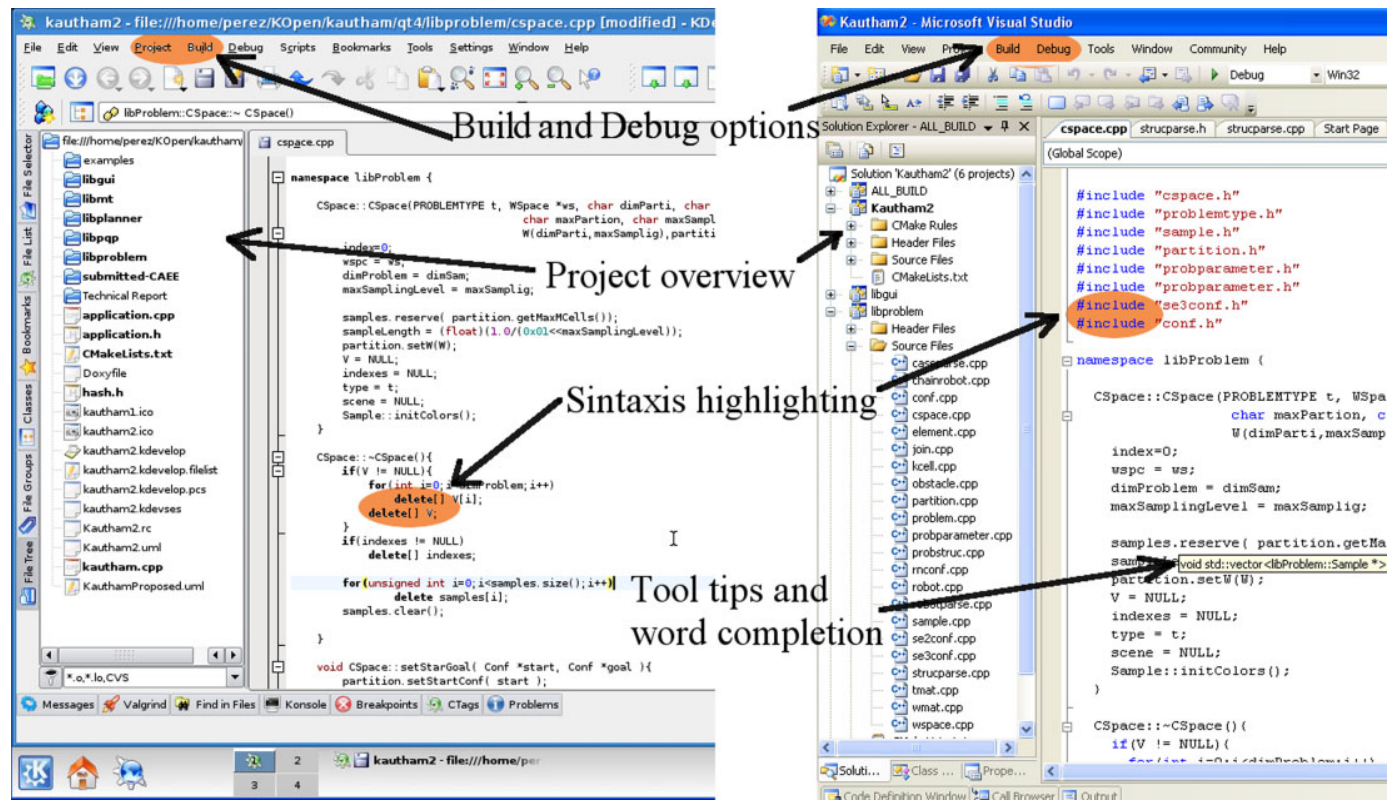

Figure 3 Integrated Developer Environment in Linux and Windows and their powerful helps. [Color figure can be viewed in the online issue, which is available at www.interscience.wiley.com.]

Several other tools could be used to make the graphical representation of three-dimensional worlds, like VTK (www.vtk.org) or the sgi OpenInventor release (oss.sgi.com/projects/inventor); but Coin $3 D$ has been used in many commercial as well as opensource projects and it has an active and efficient support list.

GUIs. Usually, any software application has a GUI to interchange information with the user. Several libraries can be used to build GUIs, some of them being platform specific like $M F C$ in Windows. Nevertheless, if a multi-platform application is desired, it is necessary to use the same library in all OS. In this direction, several graphical windows systems initially born in Linux environments have migrated to Windows or Mac OS's, like GTK+ (www.gtk.org), wxWidgets (www.wxwidgets.org), FOX (http://www.fox-toolkit.com) or the $Q t$ library (http://trolltech.com/products/qt).

The $Q t$ library is currently used in a great variety of open-source projects and commercial applications

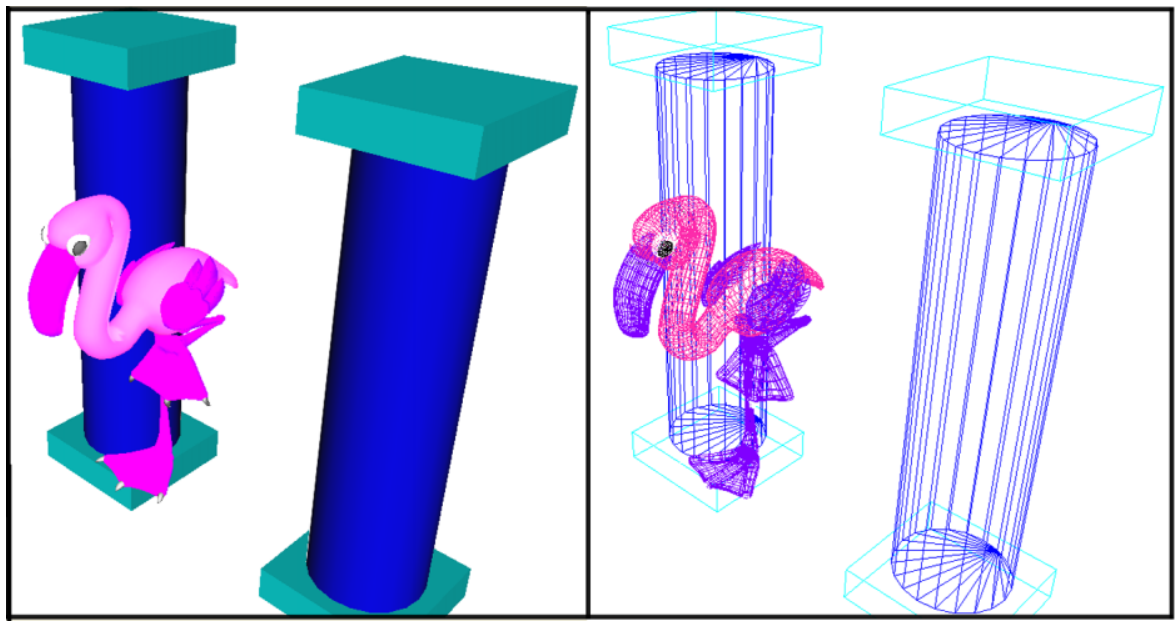

Figure 4 Three-dimensional models described using Coin3D. This figure shows the difference between primitive solids (cubes and cylinders) and triangular meshes. [Color figure can be viewed in the online issue, which is available at www.interscience.wiley.com.] 


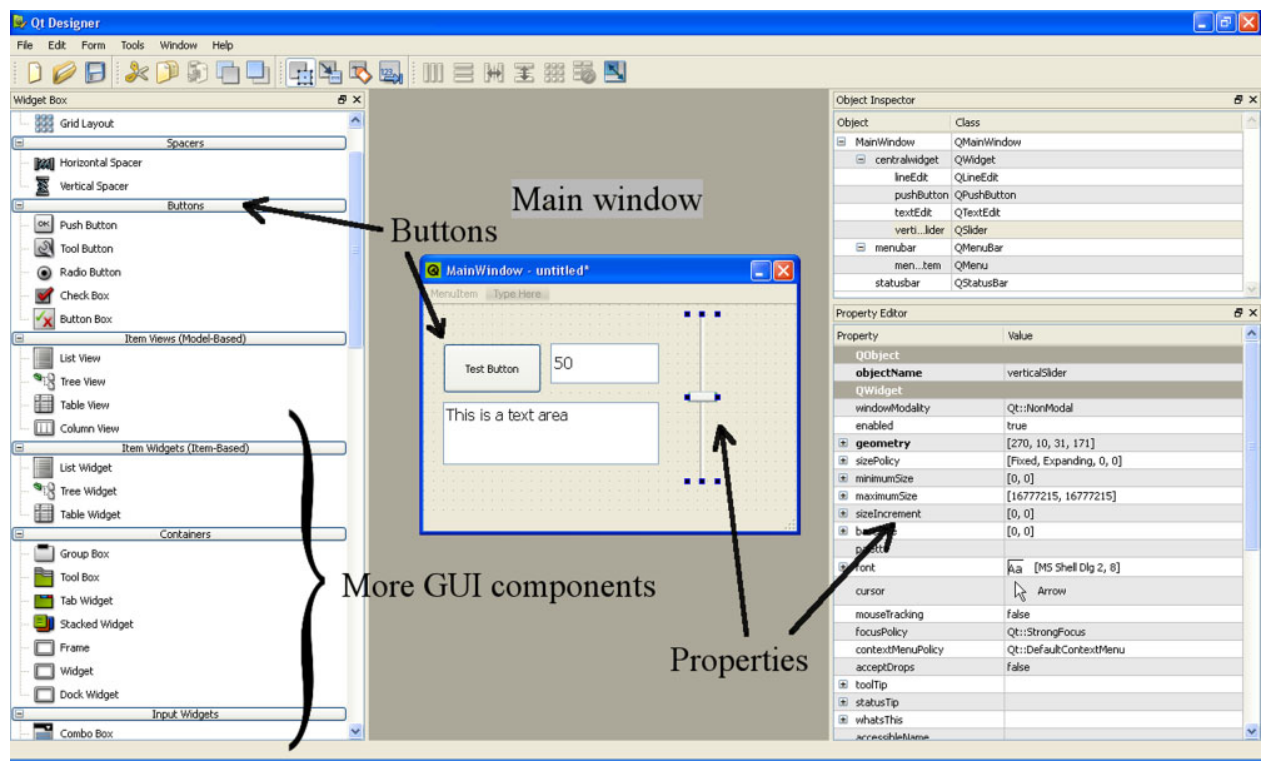

Figure 5 QtDesigner snapshot. This facility provides every common component used in a GUI. [Color figure can be viewed in the online issue, which is available at www. interscience.wiley.com.]

and it has more than 10 years of developing experience and it can easily be integrated with Coin3D. Moreover, $Q t$ has a friendly GUI designer tool (QtDesigner) based on drag and drop that helps in this creative process (Fig. 5).

To build a GUI with a $\operatorname{Coin} 3 D$ scene a $S o Q t$ library is required. This library must be properly compiled for a specific $Q t$ and Coin $3 D$ version.

The $Q t$ library is not only a graphic objects set, but a complete library that cover many topics of the software developing process, for example, it provides components for XML processing, string manipulation, file reading and much more.

Qt has one particularity that makes it different from others GUI frameworks: the signal and slots technology used for the communication between objects. It is a safer alternative to the use of the callback functions technique. The objects do not need knowledge about each other to communicate; the signals and the slots allow for a loosely coupled programming. In the build process, this characteristic entails an extra process for any object that uses it. $Q t$ calls this role Meta-Object System and the library provides the tool called moc for processing any object that use it. See Blanchette and Summerfield [11] and online documentation.

Practical Setup. To obtain a GUI with all the common functionalities like toolbars, menus, frames, tabs an so on, together with a three dimensional space, a correct interaction between the
Coin3D and $Q t$ libraries is needed using the small "glue" library SoQt. This library provides all the necessary elements to show a Coin $3 D$ window inside any $Q t$ container like panels or frames.

These three libraries (Coin3D, Qt, and SoQt) must be compiled in the same compiler, for example, gcc or $c l$. In order to properly configure the developing environment these steps must be followed:

(a) Create the system variables $\$(Q T D I R)$ and $\$(C O I N 3 D)$.

(b) Download the Coin3D library and decompress it in the $\$(\operatorname{COIN} 3 D)$ folder.

(c) Go to the build folder in Windows or the src folder in Linux and build all the libraries included.

(d) Install the latest build libraries and header files associated to the $\$(C O I N 3 D)$ folder through "make install" in Linux or through bat files in Windows.

(e) Download the Qt4 open source library and decompress in $\$(Q T D I R)$ folder. If the operating system is Windows you need to patch it to be used with the $c l$ compiler (See additional information in http://psi-im.org/ wiki/Compiling_Qt4_on_Windows).

(f) Configure Qt4 library using the qconfigure utility in Linux or using qconfigure.bat in Windows.

(g) Build the library using make in Linux or nmake in Windows. 
(h) Download SoQt and decompress it in a folder.

(i) Go to the build folder in Windows or the src folder in Linux and build all the libraries included.

(j) Install the latest build libraries and header files associated to the $\$(Q T D I R)$ folder through "make install" in Linux or through bat files in Windows.

Now the framework has the capabilities to compile and show a three-dimensional window inside any $Q W i d g e t$ container like the QTabWidget shown in Figure 6, through invoking the SoQtExaminerViewer constructor. A data structure Viewer is shown in the same figure and it groups several $\operatorname{Coin} 3 D$ data types.

\section{Computational Geometry Tools}

Collision Detection. An important part of a motion planner is the collision check process that verifies if configurations are free or not. Several open-source libraries are available on the internet. They use different techniques, like Oriented Bounding Box [12], Axis-Aligned Bounding Box [13], or Swept Spheres Volumes [14], each one with its own advantages and disadvantages. A good alternative is the Proximity Query Package (www.cs.unc.edu/geom/ $\mathrm{SSV}$ ) in forward $P Q P$. There are many applications using it since it is based on quick and reliable

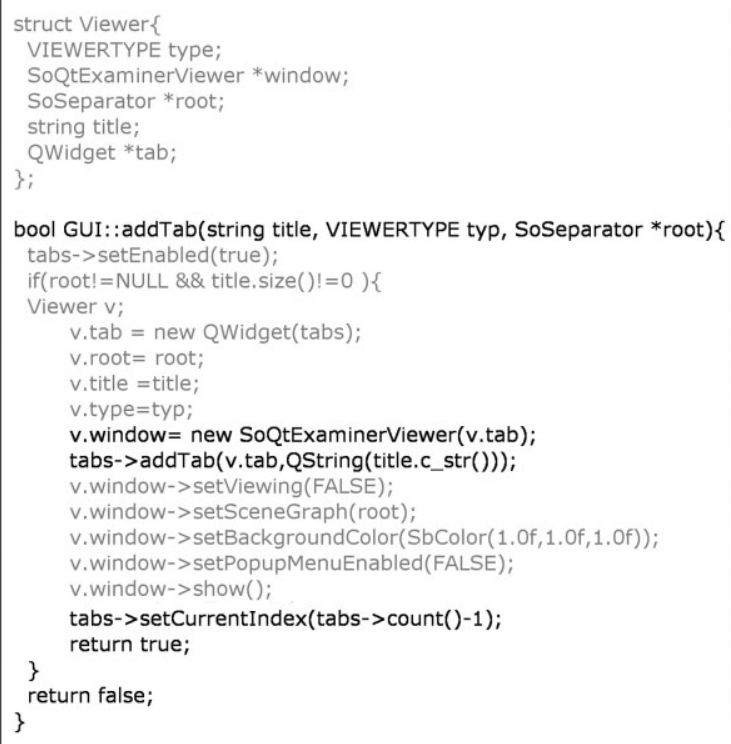

Figure 6 Adding a 3D viewer into a tab control in Qt4. The most important instructions are showed in dark and they are used to create the viewer, to push it into the tab container and bring it to the front. algorithms to find collisions between objects described by triangular meshes, and also it can be asked for the separation distances between models.

For rendering purposes $P Q P$ must be connected to a graphics library like Coin $3 D$, since $P Q P$ does not have any graphics components. $P Q P$ models are based on triangular meshes. These triangular meshes can be obtained from VRML or Inventor models using a conversion procedure provided by the Coin $3 D$ library (Fig. 7).

Motion Planning Tools. Currently, sampling-based methods are the most commonly used methods in motion planning problems. These methods consist in the generation of collision-free samples of configuration space (Cspace) and in their interconnection with free paths, forming either roadmaps (PRM [5]) or trees (RRT [6]). PRM planners are conceived as multi-query planners, while $R R T$ planners are developed to rapidly solve a singlequery problem. Some of the needs to develop a sampling-based motion planner are: (a) the generation of samples, (b) the search of nearest neighbors, (c) graph representations and search algorithms.

Samples can be generated using either a random or a deterministic sequence. Random samples are

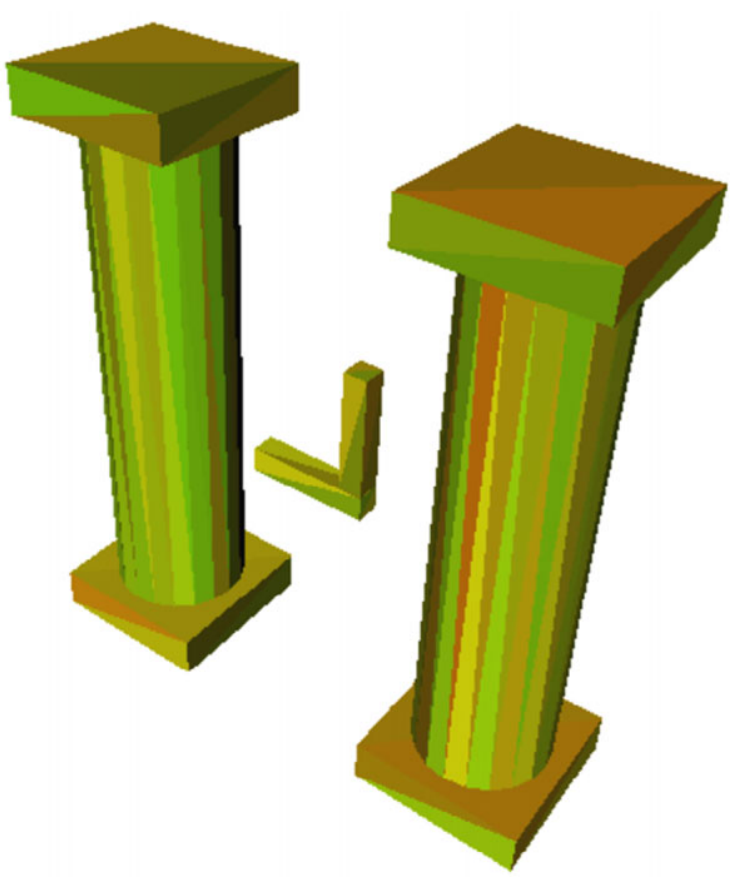

Figure 7 PQP model graphically represented as a Coin3D triangular mesh. The PQP model has been obtained from several Coin3D primitives (cubes and cylinders). [Color figure can be viewed in the online issue, which is available at www.interscience.wiley.com.] 
generated by randomly setting the values of the configuration coordinates. These values are usually obtained using a pseudo-random number generator with long period and good statistical acceptance, like the one provided by Rabin [15] that gives better results than the rand function of the standard $\mathrm{C}$ library. Deterministic sequences provide samples giving a good incremental and uniform coverage of Cspace. The Halton sequence is one of the best low-discrepancy sequences (http://people.scs.fsu.edu/ burkardt, [16]). Some of these sequences can also provide a lattice structure to easily allow the determination of the neighborhood relations, like the $S d k$ sequence [17] that is based on a multi-grid cell decomposition with an efficient cell coding, and on the use of the digital construction method.

In order to connect samples to capture the connectivity of the free configuration space, it is necessary to find, for each sample, which are its nearest neighbors. This can be efficiently solved using the ANN library (www.cs.umd.edu/mount/ANN, [18]). $A N N$ is a library written in $\mathrm{C}++$, which supports data structures and algorithms for both exact and approximate nearest neighbor searching in arbitrarily high dimensions. A set $P$ of input samples are preprocessed into a $k d$-tree data structure so that given any query sample $q$, the nearest or generally $k$ nearest samples of $P$ to $q$ can be reported efficiently. The distance between two samples can be defined in many ways. $A N N$ assumes that distances are measured using any class of distance functions called Minkowski metrics. These include the well known Euclidean distance, Manhattan distance, and max distance. An adaptation of the ANN library to different topologies of Cspaces, with its corresponding metrics is available at msl.cs.uiuc.edu/yershova, [19].

To deal with graph data structures, the Boost Graph Library (www.boost.org/libs/graph, [20]) is a library implemented using the generic programming paradigm that provides a standardized generic interface with template search algorithms like Breadth First, Depth First, or Uniform Cost, and other algorithms that allows access to a graph's structure. These algorithms can easily be tailored to any particular application. The Boost Graph Library is a header-only library and does not need to be built to be used.

\section{Practical Setup}

Loading Scenes. Scenes can be defined using description files in text, $X M L, V R M L$, or Inventor code. Coin $3 D$ uses either Inventor or VRML as input files (these models of robots and obstacles can be obtained from the Internet or converted from $C A D$ models). To help the user in defining a scene, however, the $X M L$ language can be very useful. A good alternative to easy handle $X M L$ files is to use the Simple API for XML (SAX, http://sax.sourceforge.net). The $S A X$ technique defines a way to write and read every tag and their parameters in an $X M L$ file to be processed. The other main technique to handle these files is the Document Type Definition (DTD, http:// www.w3schools.com/dtd), but this is a more complex technique.

The $X M L$ files can contain information of the robot and of the obstacles (VRML or Inventor files, names, positions, and scales), and any other aspect related to the definition of a planning problem, like the initial and goal configurations. As an example, Figure 8 shows an $X M L$ file that with the following information: the problem's type is R6 (meaning that the Cspace is that of a kinematic chain with six links), the workspace represented in the Inventor file columns.iv with a scale factor of 1.8 , the robot described in the Inventor file rtx90.iv with a scale factor of 0.7 , and the start and goal joint values. Any extra parameter needed can be easily added by defining the corresponding tags.

$Q t$ has an $X M L$ module based on the $S A X$ technique. It has an abstract class named QXmlDefaultHandler that can be quickly tailored to any file structure. Only two main functions need to be implemented, called startElement and endElement, to process each defined element in the input $X M L$ file. Connecting PQP With Coin3D. $P Q P$ performs collision checking using its own models, based on triangular meshes, that are usually build from the corresponding Inventor ones. In order to efficiently use these models, a higher level class can be first defined to represent the model of any 3D object. This class is composed by an Inventor model used for visualization purpose, and a $P Q P$ model used for computational geometry purposes (collision or distance check). Also, the class must have

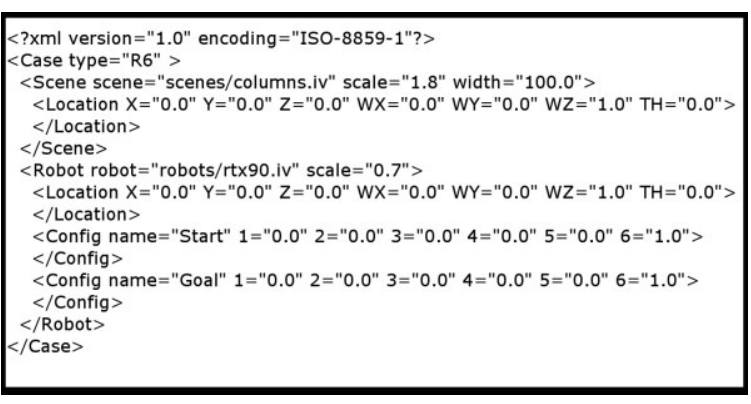

Figure 8 XML file describing a scene. 
bool Element::makePQPModel()

if(this->ivmodel != NULL) \{

this-> pqpmodel $=$ new PQP Model ;

SoCallbackAction triAction;

tri_info info(pqpmodel,scale);

pqpmodel->BeginModel();

triAction.addTriangleCallback(SoShape: :getClassTypeId()

triAction.apply(ivmodel) (void*)\&info)

pqpmodel->EndModel();

return true;

return false;

Figure 9 A possible implementation of a method to obtain the PQP model from the Inventor one. Shown in dark the main Coin3D functions used to extract the triangular mesh from the primitive solids.

procedures to convert from one model to the other. As an example, Figure 9 shows the conversion function between models of a class of this type, called Element. This function makes a $P Q P$ model from an Inventor model.

The function shown in Figure 9 uses several tools offered by Coin $3 D$ in order to extract points from any basic primitive (e.g., cylinders, cubes,...) or from any three-dimensional object, and group them in triangles. This set of triangles conform the $P Q P$ model. Since collision queries need to be answered as quick as possible, the best alternative is to make this conversion of models only once and then, for each query required, explicitly pass the position and orientation of the robot as a parameter of the query function. Care must be taken with Coin3D-PQP spatial transformations, because $P Q P$ and Coin $3 D$ use a different mathematical representation for the transformation matrix and this can give many troubles if not correctly handled (see the online documentation in www. coin3d.org).

\section{KICK-OFF EXAMPLES}

This section first presents some useful tips to prepare a project using CMake and then proposes three examples. The code can be downloaded from http://iocnet.upc.edu//usuaris/JanRosell/EducationalTools.html; it includes a Readme file with instructions to generate the executable files. These examples illustrate how to load a scene and show it using Coin3D, how to convert from Coin $3 D$ models to $P Q P$ models and test for collisions, and finally how to sample a configuration space. All these are basic needs in the development of a planner. The complete documentation of the classes used in these examples can be obtained from the code using the Doxygen documentation system.

\section{Preparing a Project}

The CMake utility allows the user to prepare the project in both Windows and Linux, relieving him from the task of configuring the project for each platform. This helps the applications to be really multi-platform since the projects for compiling and linking them are easily obtained for each platform from the instructions coded in a text file (CMakeList.txt) using a simple script language. An example of a CMakeList file that generates an executable is the following:

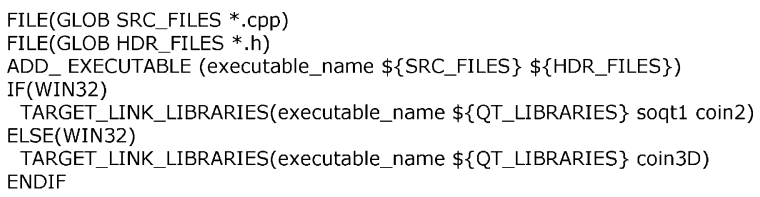

The first two lines indicates where to find all *.cpp and *.h files that have to be included in the project. The third line makes the executable and the last five lines link it with Coin3D, SoQt, and $Q t$ libraries. This linking process depends on the OS and the CMake provides the convenient tools to choose the correct modifiers in each situation.

CMake has many modules to search and configure specific libraries like Qt4, to avoid possible errors during the configuration process:

FIND_PACKAGE(Qt4 REQUIRED)

These modules assume some defaults setup values in the configuration of these libraries that have to be kept in mind to avoid passing the wrong values to the compiler or linker (if different values from the default ones are desired they have to be explicitly set). For instance, if the use of the $X M L$ processing module from Qt4 is required, you should add the following two lines in the CMakeLists file because otherwise it is turned off by default:

SET( QT_USE_QTXML TRUE )

INCLUDE_DIRECTORIES( $\left.\$\left\{Q T \_Q T X M L \_I N C L U D E \_D I R\right\}\right)$

When these modules are not found, like with the Coin3D library, all the required values must be explicitly set for the compiler and the linker, although with some experience it is possible to program a module to search and configure any library.

One feature of Coin $3 D$ is the use of signals and slots technique. This particularity causes an extra 
processing for any objects that use it, that CMake handles with the following lines:

QT4_WRAP_UI( QTUI_H_SRC myinterface.ui ) QT4_WRAP_CPP(QT_MOC_SRC gui.h)

ADD_EXECUTABLE (executable_name $\$\{$ SRC_FILES $\}$ $\$\{$ HDR_FILES $\}$ \$ $\{$ QT_MOC_SRC $\}$ \$ $\{$ QTUI_H_SRC $\}$ )

The first line preprocesses the Graphical User Interface created with the QtDesigner, for example, file myinterface.ui, and creates a header file called ui_myinterface.h referenced with the variable QTUI_H_SRC. The second line deals with the extra step, called moc or Meta-Object Compilation, that generates, using the file gui.h where the slots are defined, the source file moc_gui.cxx referenced by the variable QT_MOC_SRC. At the third line, both QTUI_H_SRC and QT_MOC_SRC are added to the source files used to create the executable.

Another useful tip for the Windows environment is the following. When a GUI application based on $Q t$ is running on Windows, a command window always appears. If this is not desired (no output to the standard output is required), the Qt4 module called QtMain should be used instead of the API winmain function. In order to correctly generate a WIN32 executable, be careful to add the following lines to the CMakeList file.

SET( QT_USE_QTMAIN TRUE)

ADD_EXECUTABLE(executable_name WIN32 \$\{SRC_FILES $\}$...

The CMakeLists.txt files of the following examples can serve as a first approach to the subject. More information about how to configure, run and use the CMake application can be read from the book Mastering CMake [10] or directly from the application help.

\section{Loading a Scene}

Loading a scene and defining the problem is the first need to be faced. The robot, the obstacles and the limits of the workspace are to be introduced to properly show the problem scene. This information can be given in an $X M L$ file. This example uses a class named StrucParse to extract and process this information. For the modeling, a class named Element is defined as the abstraction of any tree-dimensional object to be introduced in the scene. The geometry of the objects is given in either Inventor or VRML files. Figure 10 shows the snapshot of the scene loaded, where an L-shaped free-flying object is located among two obstacle columns.

\section{Testing PQP}

This example shows how to create the $P Q P$ models from Coin $3 D$ models and how to make collision

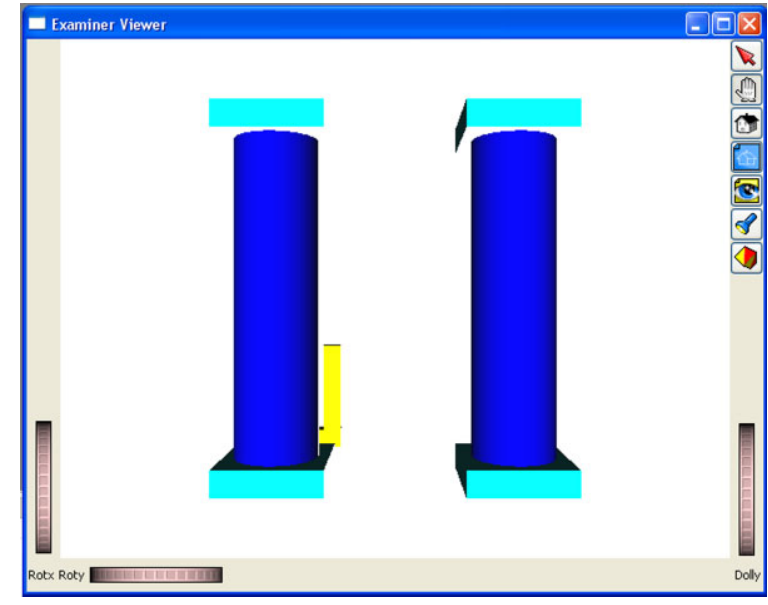

Figure 10 GUI of loading scene example. [Color figure can be viewed in the online issue, which is available at www.interscience.wiley.com.]

queries. It also serves as example to the generation of a GUI that includes sliders, buttons, tabs and 3D scenes. To execute this example it is necessary to first obtain and compile the $P Q P$ library properly. Figure 11 shows a snapshot of the GUI; it contains two tabs with $\operatorname{Coin} 3 D$ scenes, the first one with the original Inventor scene, the other one showing the $P Q P$ model where the triangular mesh is highlighted.

\section{Sampling Cspace}

This example illustrates how to sample a $d$-dimensional hypercube using random and deterministic

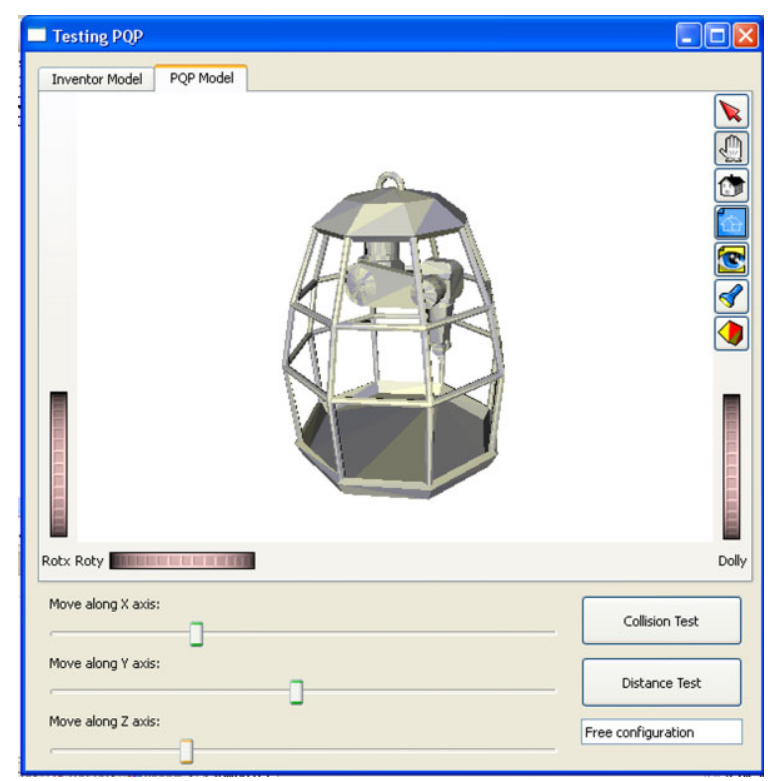

Figure 11 GUI of Testing PQP example. [Color figure can be viewed in the online issue, which is available at www.interscience.wiley.com.] 


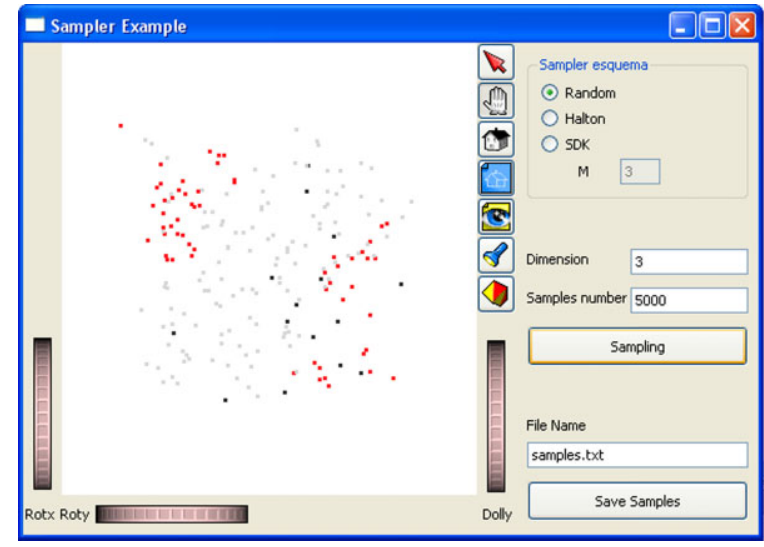

Figure 12 GUI of sampling space example. [Color figure can be viewed in the online issue, which is available at www.interscience.wiley.com.]

sampling strategies (Halton and $s d k$ ). An output text file can be written with the sample coordinates, and for $d=2$ and $d=3$ the samples are also graphically shown. Figure 12 shows a snapshot of the example GUI.

\section{CONCLUSIONS}

This paper has introduced a framework for the implementation of a motion planner under an opensource multi-platform philosophy. First, some general development tools are suggested to aid the generation of a software project. They include the programming paradigm $(O O P)$, the programming language $(\mathrm{C}++)$, a control version system $($ OpenSVN), software design tools $(\operatorname{Star} U M L)$, a multi-platform project generation tool (CMake) and a documentation tool (Doxygen). Then, graphics issues are discusses and $Q t$ is proposed as a graphical user interface library and $\operatorname{Coin} 3 D$ as the library for the graphical representation of 3D worlds. The framework finally includes proposals for the computational geometry needs: collision detection software $(P Q P)$ and motion planning tools like sampling (random, Halton, Sdk), nearest neighbor computation $(A N N)$, and graph representation and searching (Boost graph library).

As a didactic guide towards the final goal of implementing a motion planner, the paper ends providing some kick-off examples that illustrate: how to load a scene (that represents the workspace and the robot) described by an $X M L$ file and an Inventor file; how to use the collision detection package $P Q P$ together with the visualization in Coin $3 D$; and how to saple a configuration space using both random and deterministic sampling sequences
(Halton and $S d k$ ). These examples aim at facilitating the students' start-up.

The framework is currently being used by the authors as a teaching aid in a post-graduate motion planning course (some practical exercises to create a basic motion planner based on a probabilistic roadmap can be downloaded together with the kick-off examples). The framework has also been used as the base of the Kautham Planner, a motion planner based on deterministic sampling and harmonic functions developed by the authors [21].

\section{ACKNOWLEDGMENTS}

This work was partially supported by the CICYT projects DPI2008-02448 and DPI2007-63665.

\section{REFERENCES}

[1] J. C. Latombe, Robot motion planning, Kluwer Academic Publisher, New York, 1991.

[2] S. M. LaValle, Planning algorithms, Cambridge University Press, New York, 2006.

[3] L. E. Kavraki and S. M. LaValle, Handbook of robotics, Springer-Verlag, Berlin Heidelberg, 2008, pp 109-129.

[4] E. Plaku, K. E. Bekris, and L. E. Kavraki, OOPS for planning: An online open-source programming system, IEEE Int Conf Robotics Autom (2007), 3711-3716.

[5] L. Kavraki and J.-C. Latombe, Randomized preprocessing of configuration for fast path planning, IEEE Int Conf Robotics Autom (1994), 2138-2145.

[6] J. J. Kuffner Jr., and S. M. LaValle, RRT-Connect: An efficient approach to single-query path planning, IEEE Int Conf Robotics Autom (2000), 995-1001.

[7] G. Booch, Object-oriented analysis and design with applications, Addison Wesley Longman Publishing Co., Inc., Redwood City, CA, 2004.

[8] G. Booch, J. Rumbaugh, and I. Jacobson, The unified modeling language. Addison Wesley, Massachusetts, 1999.

[9] B. Collins-Sussman, B. Fitzpatrick, and M. Pilato, Version control with subversion. O'Reilly Media Inc., Sebastopol, CA, 2007.

[10] K. Martin and B. Hoffman, Mastering CMake: A cross-platform build system. Kitware Inc., New York, 2005.

[11] J. Blanchette and M. Summerfield, C++ GUI programming with Qt 4. Prentice Hall PTR, Upper Saddle River, NJ, 2006.

[12] S. Gottschalk, M. C. Lin and D. Manocha, OBBTree: A hierarchical structure for rapid interference detection, Computer Graphics 30 (1996), 171-180. 
[13] G. van den Bergen, Efficient collision detection of complex deformable models using AABB trees, J Graph Tools 2 (1997), 1-13.

[14] E. Larsen, S. Gottschalk, M. C. Lin, and D. Manocha, Fast proximity queries with swept sphere volumes, Department of Computer Science, University of North Carolina, Chapel Hill, 1999.

[15] S. Rabin, AI game programmingn wisdom 2. Charles River Media Inc., Hingham, Massachusetts, 2003.

[16] J. H. Halton, On the efficiency of certain quasi-random sequences of points in evaluating multi-dimensional integrals, Numer Math 2 (1960), 84-90.

[17] J. Rosell, M. Roa, A. Pérez, and F. García, A general deterministic sequence for sampling d-dimensional configuration spaces, J Intell Robotic Syst 50 (2007), $361-373$.

\section{BIOGRAPHIES}

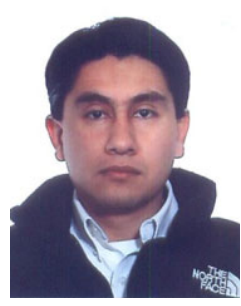

Alexander Pérez was born in Bogota, Colombia, in 1975. He is a Mechanical Engineer from the National University of Colombia (1999), and MSc in Electronic and Computer Engineering from Los Andes University (Bogotá, Colombia) in 2003. He has worked as Project Engineer and he was auxiliary professor at the National University of Colombia and the University New Granada. Since 2004 he is Assistant professor in the Electronic Engineering Faculty of the "Escuela Colombiana de Ingeniería "Julio Garavito" placed in Bogotá, Colombia. Presently, $\mathrm{He}$ is researching in the robot motion planning area inside of Institute of Industrial and Control Engineering.
[18] S. Arya, D. M. Mount, N. S. Netanyahu, R. Silverman, and $\mathrm{A}$. Y. Wu, An optimal algorithm for approximate nearest neighbor searching fixed dimensions, J ACM 45 (1998), 891-923.

[19] A. Yershova and S. LaValle, Improving motionplanning algorithms by efficient nearest-neighbor searching, IEEE Trans Robotics 23 (2007), 151157.

[20] L.-Q. Lee, J. G. Siek, and A. Lumsdaine, The generic graph component library, 14th ACM SIGPLAN conference on Object-oriented programming, systems, languages, and applications (1999), 399-414.

[21] J. Rosell, C. Vázquez, A. Pérez, and P. Iñiguez, Motion planning for haptic guidance, J Intell Robotic Syst 53 (2008), 223-245.

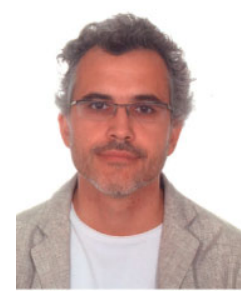

Jan Rosell received the BS degree in Telecomunication Engineering and the $\mathrm{PhD}$ degree in Advanced Automation and Robotics from the Technical University of Catalonia, Barcelona, Spain, in 1989 and 1998, respectively. He joined the Institute of Industrial and Control Engineering in 1992 where he has developed research activities in robotics. He has been involved in teaching activities in Automatic Control and Robotics as Assistant Professor since 1996 and as Associate Professor since 2001. His current technical areas include robot motion planning, haptics, robotic assembly and manufacturing automation. 